\title{
An Analysis of the Network Selection Problem for Heterogeneous Environments with User-Operator Joint Satisfaction and Multi-RAT Transmission
}

\author{
J. J. Escudero-Garzás and C. Bousoño-Calzón \\ Department of Signal Theory and Communications, University Carlos III de Madrid, Leganés, 28911 Madrid, Spain
}

Correspondence should be addressed to J. J. Escudero-Garzás; jescugar@tsc.uc3m.es

Received 4 August 2016; Revised 19 November 2016; Accepted 20 December 2016; Published 18 January 2017

Academic Editor: Luca Reggiani

Copyright (C) 2017 J. J. Escudero-Garzás and C. Bousoño-Calzón. This is an open access article distributed under the Creative Commons Attribution License, which permits unrestricted use, distribution, and reproduction in any medium, provided the original work is properly cited.

\begin{abstract}
The trend in wireless networks is that several wireless radio access technologies (RATs) coexist in the same area, forming heterogeneous networks in which the users may connect to any of the available RATs. The problem of associating a user to the most suitable RAT, known as network selection problem (NSP), is of capital importance for the satisfaction of the users in these emerging environments. However, also the satisfaction of the operator is important in this scenario. In this work, we propose that a connection may be served by more than one RAT by using multi-RAT terminals. We formulate the NSP with multiple RAT association based on utility functions that take into consideration both user's satisfaction and provider's satisfaction. As users are characterized according to their expected quality of service, our results exhaustively analyze the influence of the user's profile, along with the network topology and the type of applications served.
\end{abstract}

\section{Introduction and Motivation}

In the realm of wireless communications, a user has nowadays different possibilities of connectivity: 3G, 4G, WiFi, and WiMAX, among others. Very often, the access points (APs) or base stations (BSs) that provide these radio access technologies (RATs) coexist in the same location and, consequently, their connectivity services overlap. This scenario is known as heterogeneous network (HetNet) and has received an increasing attention in the past years $[1,2]$. On the other hand, user's satisfaction perceived when he/she is using any application is directly related to the quality of service (QoS), which is consequently a decisive factor for successful network exploitation [3-5]. Within this paradigm, we are interested in the network (or RAT) selection problem (NSP) with QoS, in which each terminal (user) is assigned to the most suitable available network complying with the QoS requirements.

In the recent literature, we can find different approaches to address the network selection problem that consider QoS constraints [6-14]. Most of these works are focused on fulfilling user's satisfaction $[7,8,10,12,14]$ (see also references in [15]). Besides, some of these works consider a singleuser scenario [7, 10,12], which is unrealistic. However, this perspective disregards operator's satisfaction, which owns and manages the network to make a profit. This networkcentric approach is not frequent in the literature and has been adopted, for instance, in [16-19].

A more complete analysis is to contemplate both user's and operator's satisfaction in multiuser scenarios, as in $[9,11$, $13,20]$. Nevertheless, this approach has received much less attention than those mentioned above. In [9], the authors address the NSP although they do not use a unified framework as they study user's and operator's satisfaction by means of two separate algorithms. In [11], the NSP is formulated as the maximization of an objective function that includes the user's and operator's utility function, and the results show the sum-utility achieved. In [13], user's and operator's satisfaction is based on monetary criteria (bid and price). However, this 
scheme relies on the so-called "operator's reputation rating," which is provided by the operator itself and may be a source of unreliability. In [20], new metrics are proposed to evaluate user's satisfaction and system performance, for multicast transmissions.

A common feature to all the abovementioned works is that the user terminal associates with only one RAT/AP. Recently, the multi-RAT technique has been proposed for heterogeneous scenarios so that the user equipment can transmit/receive its data over multiple RAT/AP [21, 22]. These terminals are known as multimode terminals (MMT) [23]. Additionally, multi-RAT parallel transmission from more than one BS/AP naturally achieves some degree of load balancing at the expense of a theoretical increase in complexity. In this paper, we contemplate the possibility that MMT has the capability to support multi-RAT parallel downlink communication (see, e.g., $[21,24]$ ).

In this work, we incorporate the use of multi-RAT transmission to the scenario where both user's and operator's satisfaction are explicitly considered. In this context, we investigate how different user's profiles may affect the performance of the system, not only in terms of utility but also in terms of metrics significant for heterogeneous networks such as bit rate and BS load. The NSP is solved following three different approaches to achieve some degree of load balancing in a natural way, so the use of specific convex/concave utility functions can be avoided [25]. Among the references mentioned above, this work is closer to [11]. Nevertheless, we here consider multi-RAT transmission and explore explicit metric and results that show user's and operator's satisfaction, different performance according to user's profile distribution, and different performance for distinct network topologies.

The rest of this paper is organized as follows. Section 2 provides a review of the related literature and distinguishes the most usual approaches, multiobjective optimization, and game theory. In Section 3, we present the system model, which includes user's characterization and the definition of utility functions. In Section 4, we provide the mathematical formulation for the NSP. Performance evaluation of the developed options is presented and analyzed in Section 5. The paper ends with our conclusions.

\section{Related Work}

Network selection concerning users and providers for HetNets have been examined mainly from two viewpoints, namely, multiobjective optimization and game theory. While the multiobjective functions allow for different (and frequently opposite) criteria of interest for the actors of the network (base station, access point, operator, and users), game theory resolves the conflicting interests under a selfish perspective of the participants. In this section, we explore the relevant literature in these two areas.

2.1. Multiobjective Optimization for NSP. Multiobjective optimization (also known as multicriteria optimization) is a mathematical framework to solve problems with multiple objectives or criteria. As in multiobjective optimization (MOO), the objectives are incorporated into the objective function, the results are more aligned with the objectives than when these objectives are formulated as constraints in the optimization problem. MOO has been applied in many engineering and economic related fields and is receiving an increasing attention to study wireless communication problems with opposed criteria [26].

In [9], the NSP is formulated as the maximization of a new multiobjective function that includes several criteria that collect both user preferences and network status (link quality, cost, battery lifetime, mobile speed, and network load). Instead of constructing the multiobjective function as the weighted sum of the individual criteria utilities, the authors propose an exponential-weighted product utility function, as they consider that if given criteria are set to zero, the total utility must reflect the importance of this fact. They also propose how to integrate the mechanism into the MIH (Media-Independent Handover) mechanism defined in IEEE 802.11 standard. However, it is difficult to determine the benefits of this work as user's and operator's satisfaction are evaluated separately.

In their paper, Kosmides et al. [11] address the NSP from a service utility-based perspective, as the user's and operator's utility are a function of the provided service (voice, video, or web). As video and web are considered soft services, in the sense that different rates can be accommodated, the utility function of these two services has two components, the user's utility and the operator's utility. Also, the contribution of each component is complementary weighted by using the $w$ parameter, with $w \in[0,1]$. In this model, users are characterized depending on their expected satisfaction level, and this characterization conditions the operator's utility in the form of user's willingness to pay. To solve the NSP, the authors propose two heuristic algorithms, the Greedy algorithm and the strip packing algorithm. Having the same $\mathcal{O}\left(N M^{2}\right)$ complexity ( $N$ is the number of access points and $M$ is the number of users), the two algorithms perform differently depending on the chosen metric.

In [27], the authors propose two models that include battery consumption, quality of service, and monetary cost. The first model is based on TOPSIS (Technique for Order of Preference by Similarity to Ideal Solution) and incorporates a vote mechanism to reduce user's subjectivity. The second model relies on the data envelopment analysis (DEA) methodology with the goal of achieving good user's experience with no explicit requirements. This scheme accomplishes a reduction in complexity with respect to the TOPSIS scheme.

In [28], the authors propose an access network selection mechanism based on the IEEE 802.21 standard. In this model, user's utility evaluates fairness among users and the suitability of the network currently selected. Regarding provider's utility, it is based on the total income. The overall network selection process is implemented by means of a genetic algorithm.

2.2. Game-Theoretic Approach for NSP. Game theory is a mathematical tool that is suitable to model situations where 
the involved participants or players (users, base stations in our case) compete selfishly for the resources. The combination of strategies (or decisions) incorporating the best strategy for every player is known as equilibrium, which is a Nash Equilibrium if none of the players can increase its utility by changing his/her strategy without degrading the utility of the others [29]. Hence, game theory can be straightforwardly applied to NSP. For the interested reader, [30] provides an excellent survey of the literature on the general problem of network selection based on game theory previous to 2010.

In [31], the proposed game-theoretic network selection framework integrates service differentiation considering distinct utility functions for brittle, partially elastic, and elastic traffic, from the user's perspective, similarly to [11]. The objective is to maximize the sum-utility, as the sum of the user's utilities. To this end, a local improvement algorithm (LIA) is proposed. The key point of LIA is the use of localized cooperation, that is, two networks/APs whose coverage overlaps exchange information. LIA takes the form of a user-network association game in which the pairs of overlapping networks are the players and the original problem is decomposed into a sequence of multiple subproblems. However, provider's profit is not explicitly contemplated, as only social welfare is taken into consideration.

In [13], a modified version of the first-price sealed-bid auction is designed to solve the NSP. In this auction, the users buy the connection service to the network operators that provide it. The users establish their preferences by means of the desired buying price, which is based on the operator's reputation rating (level of success in delivering service according to a given QoS). As the reputation ranking is provided by the operator itself, this may be a potential source of unreliability. The operator's strategy is based on the price offered by the users, the bidding price, and a penalty according to the reputation rating, and the utility is equal to the difference between the bidding and the cost. For the case of two operators, which is a relevant case in practical systems, they find a closed form for the equilibrium bidding strategy functions,.

Following also a game-theoretic approach, Cao et al. [32] present a two-layer framework that involves both service provider's benefit and user's satisfaction. The two-layer algorithm consists in an intranetwork game where the service providers (SPs) determine their prices and rates for users, and an internetwork game for network selection, where users are associated with SPs according to the prices and rates resulting from the previous stage. The second game is addressed from a social-behavioral viewpoint, as the authors formulate the game as a hedonic game which considers preference rules to implement behavioral restrictions. In the same line, the work of [24] proposes a two-level algorithm, where in the user-level game they compete for provider's bandwidth and in the provider-level game providers establish bandwidth prices.

For HetNets that include C-RAN (cloud-radio access networks), inter-tier interference mitigation is studied in [33]. The key idea of this scheme is the use of contract-based utility functions for the base stations (macro base stations and radio remote units, RRH), with these utilities being a function of the sum-rate received by the users. A significant contribution of this paper is the consideration of non-perfect channel state information (CSI) for the contract design.

\section{System Model}

In this paper, we study the network selection problem (NSP) in heterogeneous networks, considering that one operator offers network connectivity services by means of a set of $M$ different radio access technologies (RATs) to $N$ users. We consider that user's terminals are multi-RAT (also known as multistandard or multimode) [34], that is, terminals that can connect to more than one radio technology and support connectivity in integrated heterogeneous environments. Each user reports his/her bandwidth (bit rate) requirements $R_{n \text {,max }}$ when he/she requests a connectivity service for applications such as voice or video streaming. We assume that our model targets mobile communication networks where users have a very reduced mobility such as airport hot-spots, restaurants, and cafés, where no horizontal handover management is required.

The operator offers connectivity services to the users from a finite set of data rates $\mathscr{R}=\left\{R_{1}, \ldots, R_{J}\right\}$; that is, the solution to the NSP is to optimally assign elements from $\mathscr{R}$ to users. As each RAT has distinct characteristics, we have $\mathscr{R}_{m}=\left\{R_{m 1}, \ldots, R_{m J_{m}}\right\}$ for each RAT $m$. We denote by $C_{m}$ the maximum bit rate that RAT $m$ can deliver to users.

3.1. User Profiles. For variable bit rate services (web and video), different user profiles are considered according to his/her expectation about the quality of service (QoS) he/she will receive and the benefit the provider may obtain for the service [11]. Some users have low expectations about the QoS, which is the case of users with low-quality service contracts or not worried about the quality of the service. These users are labeled as low-expectation users (LEUs). On the other hand, other users that are paying for high quality services are generally not tolerant to service degradation and can be characterized as high-expectation users (HEUs). Finally, the rest of users having expectations between these two cases are considered as mediumexpectation users (MEUs). The utility functions corresponding to the different profiles are presented in the following subsection.

For on-off services such as voice, where the service is provided only if the bit rate exceeds a given threshold rate, the utility function is the same irrespective of the user profile, as indicated also in the following subsection.

3.2. Utility Functions. In this section, we introduce the utility functions to be used, defined in [11]. Let $u_{s}\left(R_{m, n}, R_{n, \max }\right)$ denote the user's satisfaction function and $u_{p}\left(R_{m, n}, R_{n}\right)$ the RAT's benefit function, where $R_{m, n}$ represents the rate assigned by RAT $m$ to user $n$ and $R_{n}$ stands for the data rate requested by user $n$. Three different utility functions based on 
$u_{s}$ and $u_{p}$ are defined whether the service requested by user $n$ is either voice, web, or video:

$$
U_{\text {voice }}\left(R_{m, n}, R_{n}\right)= \begin{cases}0 & \text { if } R_{m, n}<R_{n} \\ u_{\max } & \text { if } R_{m, n} \geqslant R_{n}\end{cases}
$$

$$
\begin{aligned}
& U_{\text {web } / \text { video }}\left(R_{m, n}, R_{n}\right) \\
& \quad= \begin{cases}w u_{s}+(1-w) u_{p} & \text { web } \\
w f\left(u_{s}\right)+(1-w) f\left(u_{p}\right) & \text { video }\end{cases}
\end{aligned}
$$

where, for a generic utility function $u, f(u)$ is defined as

$$
f(u)= \begin{cases}0 & \text { if } R_{m, n}<R_{n, \min } \\ u_{\min }+\left(1-u_{\min }\right) u\left(R_{m, n}-R_{n, \min }, R_{n}-R_{\min }\right) & \text { if } R_{m, n} \geqslant R_{n, \min }\end{cases}
$$

$R_{n \text {,min }}$ is the minimum data rate requested by user $n$ for web service, and parameter $w$ weights utility functions of (2) to favor one component over the other. Note that for $w=1$ only user's satisfaction is considered, while for $w=0$ only operator's satisfaction is pertinent. In (3), $u_{\min }$ represents the minimum utility value for video service.

Mathematically, the user profiles correspond to different utility functions for both $u_{s}$ and $u_{p}$. With respect to $u_{s}$, the general form is

$$
u_{s}=\left(\frac{R_{m, n}}{R_{n}}\right)^{\gamma}
$$

where $\gamma$ takes the values 1,2 , and 4 for LEU, MEU, and $\mathrm{HEU}$, respectively [35]. Regarding $u_{p}$, the utilities for MEU and HEU are modelled using a sigmoid function because of its mathematical properties (monotonicity, convexity) and its capacity to implement the decreasing marginal utility of user's satisfaction. For LEU, a logarithmic function reflects the desired behavior. Hence, the $u_{p}$ functions are as follows:

$$
u_{p}= \begin{cases}\alpha_{\mathrm{LEU}} \log \left(1+a R_{m, n}\right), & \text { low-expectation user (LEU) } \\ \frac{\alpha_{\mathrm{MEU}}}{1+\exp \left(\delta_{\mathrm{MEU}}\left(R_{\mathrm{MEU}}-R_{m, n}\right)\right)}, & \text { medium-expectation user (MEU) } \\ \frac{1}{1+\exp \left(\delta_{\mathrm{HEU}}\left(R_{\mathrm{HEU}}-R_{m, n}\right)\right)}, & \text { high-expectation user (HEU). }\end{cases}
$$

In (5), $\delta_{\mathrm{HEU}}$ and $\delta_{\mathrm{MEU}}$ represent the steepness of the utility curve for HEU and MEU, while $R_{\mathrm{HEU}}$ and $R_{N L U}$ determine the center of the utility curves for HEU and MEU. Note that

(i) the larger the center value, the smaller the range of $R_{n}$ where users are risk-averse;

(ii) the larger the steepness, the sharper the curve.

To meet the expected user's behavior, the utility functions (1)-(5) have been accordingly fitted using the parameter values of Table 1 .

\section{Problem Formulation}

The objective is to maximize the network total utility, which is a sum of the individual utilities of the network. Let $U_{m, n}\left(R_{m, n}, R_{n}\right)$ be the utility function for the pair $(m, n)$, being $m$ the access point index and $n$ denotes the user. Note that $U_{m, n}$ will correspond to either (1) or (2). Let $R_{m, n} \in \mathscr{R}_{m}$ be the bit rate assigned to user $n$ by RAT $m$. Let $x_{m, n}$ be the binary assignment variable associated with $(m, n)$ such that $x_{m, n}=1$ if user $n$ is assigned to RAT $m$ and 0 otherwise. If each user can be associated with one and only one RAT, NSP can be formulated as [11] 
TABLE 1: Utility functions parameters.

\begin{tabular}{llc}
\hline Parameter & Description & Value \\
\hline$R_{n}[\mathrm{kbps}]$ & Rate requested by user $n$ & 12 (voice), 512 (video), 512 (web) \\
$R_{n, \text { min }}[\mathrm{kbps}]$ & Minimum threshold rate for video utility in (3) associated with user $n$ & 0 (voice), 64 (video), 64 (web) \\
$w$ & Weight of utility function components in (2) & {$[0,1]$} \\
$\alpha_{\mathrm{LEU}}, \alpha_{\mathrm{MEU}}$ & Maximum gain for LEU and MEU in $u_{p}(5)$, respectively & $0.15,0.7$ \\
$a$ & Logarithmic utility parameter (5) & 0.05 \\
$\delta_{\mathrm{MEU}}, \delta_{\mathrm{HEU}}$ & Slope of $u_{p}(5)$, for MEU and HEU, respectively & 0.03 \\
$R_{\mathrm{MEU}}, R_{\mathrm{HEU}}[\mathrm{kbps}]$ & Parameter of $u_{p}(5)$ for MEU and HEU, respectively & $R_{k} / 3.5, R_{k} / 2$ \\
\hline
\end{tabular}

$$
\begin{aligned}
\text { NSP: } \max _{x_{m, n}} & \sum_{m=1}^{M} \sum_{n=1}^{N} U_{m, n}\left(R_{m, n}, R_{n}\right) \\
\text { s.t. } & \sum_{n=1}^{N} x_{m, n} R_{m, n} \leqslant C_{m}, \quad \forall m=1, \ldots, M \\
& \sum_{m=1}^{M} x_{m, n} R_{m, n} \leqslant R_{n}, \quad \forall n=1, \ldots, N \\
& \sum_{m=1}^{M} x_{m, n} \leq 1, \quad \forall n=1, \ldots, N \\
& x_{m, n} \in\{0,1\}, \quad \forall n=1, \ldots, N, \forall m=1, \ldots, M,
\end{aligned}
$$

where (C.1) implements the maximum capacity constraint, (C.2) implements the maximum bit rate per user constraint, and (C.3)-(C.4) implement the fact that users can only be associated with one RAT.

However, a user may be willing to simultaneously execute two or more applications (for instance, voice and web browser) and, in practical implementation, the application data flow is constrained to a single RAT. To reflect this scenario, the problem is reformulated so that a total of $K \geqslant N$ connections are attended by $M$ RATs:

$$
\begin{aligned}
\text { NSP1: } \max _{x_{m, k}} & \sum_{m=1}^{M} \sum_{k=1}^{K} U_{m, k}\left(R_{m, k}, R_{k}\right) \\
\text { s.t. } \quad & \sum_{k=1}^{K} x_{m, k} R_{m, k} \leqslant C_{m}, \quad \forall m=1, \ldots, M \\
& \sum_{m=1}^{M} x_{m, k} R_{m, k} \leqslant R_{k}, \quad \forall k=1, \ldots, K \\
& \sum_{k=1}^{N} x_{m, k} \leq 1, \quad \forall k=1, \ldots, K . \\
& x_{m, k} \in\{0,1\}, \quad \forall k=1, \ldots, K, \forall m=1, \ldots, M,
\end{aligned}
$$


where $U_{m, k}, R_{m, k}, R_{k}$, and $x_{m, k}$ are equivalent to those defined above but for the pair $(m, k)$.
As stated in Section 1, multi-RAT users (applications in this case) can be associated with more than one access point. Therefore, (NSP1) can be reformulated as

$$
\begin{aligned}
\text { NSP MultiRAT: } \max _{x_{m, k}} & \sum_{m=1}^{M} \sum_{k=1}^{K} U_{m, k}\left(R_{m, k}, R_{k}\right) \\
\text { s.t. } & \sum_{k=1}^{K} x_{m, k} R_{m, k} \leqslant C_{m}, \quad \forall m=1, \ldots, M \\
& \sum_{m=1}^{M} x_{m, k} R_{m, k} \leqslant R_{k}, \quad \forall k=1, \ldots, K \\
& \sum_{k=1}^{N} x_{m, k} \leq 1, \quad \forall k=1, \ldots, K . \\
& x_{m, k} \in[0,1], \quad \forall k=1, \ldots, K, \forall m=1, \ldots, M,
\end{aligned}
$$

where constraint (C.4) makes it possible that any application $k$ can be associated with more than one RAT. Note that (NSP1) is an integer problem. Therefore, by replacing (C.4) for (C.4a), we are performing integer constraint relaxation and (NSP1) becomes the more tractable (NSP MultiRAT) problem [36]. Moreover, it has the advantage of achieving some degree of load balancing with no additional changes.

\section{Performance Evaluation}

This section shows the performance results obtained by simulation. Our proposal is compared with a Greedy stateof-the-art algorithm [11]. In the following figures, we depict the results corresponding to

(i) optimal solution to (NSP1), labeled as OPT: The solution is obtained using a standard branch-andbound $(\mathrm{B} \& \mathrm{~B})$ algorithm, as the integer problem is linear [37],

(ii) optimal solution to (NSP MultiRAT), labeled as Multi: The NSP MultiRAT problem is linear, so a standard interior point algorithm is employed [38],

(iii) a rounded solution of (NSP MultiRAT), labeled as MultiR: The solution is obtained by rounding the NSP MultiRAT solution to the nearest integer (the rounded solution is obtained by approximating the real solution to nearest integer; e.g., if we have $x_{1, k}=$ 0.7 and $x_{2, k}=0.3$, the rounded solution is $x_{1, k}=1$, $\left.x_{2, k}=0\right)$,

(iv) the Greedy algorithm, labeled as Greedy, that solves the (NSP) problem (equivalent to NSP1).
We first describe the simulation scenario and afterwards we provide the results in terms of utility and bit rate.

5.1. Simulations Setup. This subsection describes the simulation setup. The network consists of 3 base stations (BSs) or access points whose coverage areas overlap, and each of them can correspond to a different RAT, namely, HSDPA, HSDPA+, and WiMAX 802.16e. The bit rates available for each RAT are given in Table 2. The results reflect the case when the probability of blocking is zero. This is accomplished by providing the RATs with sufficient capacity to accommodate all user demands, as the objective is to analyze the possible differences among user and service profiles and RAT combinations. The capacity of each RAT has been set to $16.8 \mathrm{Mbps}$ (WiMAX), 9.4 Mbps (HSPA+), and $6 \mathrm{Mbps}$ (HSPA), considering one sector per BS [39].

For a fair comparison, the channel model is similar to that described in [11]. The BS-terminal communication can find three conditions, namely, propitious, balanced, and ominous. In our simulations, we assume the channel condition is the same for all users and BSs. The channel model is as follows. For propitious conditions, the channel is good with a probability of $70 \%$ and poor with a probability of $30 \%$. For balanced and ominous conditions, the probabilities are, respectively, $(50,50)$ and $(30,70)$. Whether the channel is good or poor, the available transmission rate is given in Table 2.

To evaluate the influence of user's profiles, we have simulated scenarios with different percentage of user's profiles and with different percentage of application's types. Table 3 details the simulated configurations. We have also considered different combinations of RATs to assess the effect of this 
TABLE 2: Simulation parameters.

\begin{tabular}{lc}
\hline Parameter & Value \\
\hline $\mathscr{R}(\mathrm{HSPA})[\mathrm{kbps}]$ & Good channel: 12 (voice), 128 (video), 128 (web) \\
$\mathscr{R}(\mathrm{HSPA}+/ \mathrm{LTE})[\mathrm{kbps}]$ & Poor channel: 0 (voice), 32 (video), 32 (web) \\
& Good channel: 12 (voice), 384 (video), 384 (web) \\
$\mathscr{R}($ WiMAX) $[\mathrm{kbps}]$ & Poor channel: 0 (voice), 128 (video), 128 (web) \\
& Good channel: 12 (voice), 512 (video), 512 (web) \\
\hline
\end{tabular}

TABLE 3: Configurations of users and applications.

\begin{tabular}{lcc}
\hline Configuration ID & Percentage of user types (LEU/MEU/HEU) & Percentage of applications (voice/web/video) \\
\hline CONFIG1 & $(1 / 3) /(1 / 3) /(1 / 3)$ & $(1 / 3) /(1 / 3) /(1 / 3)$ \\
CONFIG2 & $20 / 20 / 60$ & $(1 / 3) /(1 / 3) /(1 / 3)$ \\
CONFIG4 & $(1 / 3) /(1 / 3) /(1 / 3)$ & $0 / 0 / 100$ (only video service) \\
CONFIG5 & $(1 / 3) /(1 / 3) /(1 / 3)$ & $0 / 100 / 0$ (only web service) \\
CONFIG6 & $20 / 20 / 60$ & $0 / 0 / 100$ (only video service) \\
CONFIG7 & $20 / 20 / 60$ & $0 / 100 / 0$ (only web service) \\
CONFIG8 & $20 / 20 / 60$ & $100 / 0 / 0$ (only voice service) \\
CONFIG9 & $(1 / 3) /(1 / 3) /(1 / 3)$ & $100 / 0 / 0$ (only voice service) \\
\hline
\end{tabular}

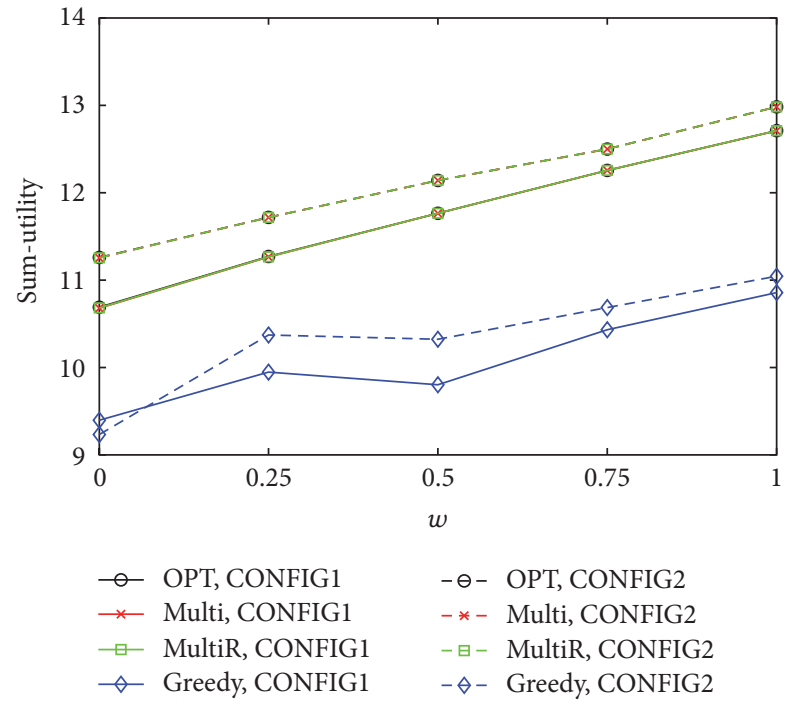

FIGURE 1: Sum-utility for users-applications configurations CONFIG1 and CONFIG2, 18 users, scenario propitious, network topology $\# 1$.

factor on the system performance. The network topologies corresponding to the RAT combinations are listed in Table 4.

5.2. Utility Performance. Figures 1 and 2 represent the variation of the sum-utility with $w$ of (2). We observe that the higher the value of $w$, the higher the sum-utility obtained, which implies that the term associated with user's utility dominates provider's utility. Regarding the percentage of user types, we notice that an increase in HEUs (CONFIG2 compared with CONFIG1 and CONFIG6 compared with



FIGURE 2: Sum-utility for users-applications configurations CONFIG4 and CONFIG6, 18 users, scenario propitious, network topology \#1.

CONFIG4) implies an increase in sum-utility keeping the same percentage of applications.

In Figures 3 and 4, the sum-utility is plotted for different combinations of BSs, as indicated in Table 4 . As expected, the sum-utility grows as the capacity offered by the set of base stations does. We note that, for the network topologies with highest sum-capacity (combinations 1 to 6), the presence of a higher percentage of high-expectation users (CONFIG2 and CONFIG6) increases the sum-utility, and this effect is almost 
TABLE 4: Network topology: number of BSs of each type.

\begin{tabular}{lcccc}
\hline Network topology ID & HSPA+ & WiMAX & HSPA & Offered sum-capacity (Mbps) \\
\hline 1 & 0 & 3 & 0 & 50.4 \\
2 & 1 & 2 & 0 & 43.2 \\
3 & 0 & 2 & 1 & 39.6 \\
4 & 2 & 1 & 0 & 36 \\
5 & 1 & 1 & 1 & 32.4 \\
6 & 3 & 0 & 0 & 28.8 \\
7 & 0 & 1 & 2 & 28.8 \\
8 & 2 & 1 & 0 & 25.2 \\
9 & 1 & 0 & 2 & 21.6 \\
10 & 0 & 0 & 3 & 18 \\
\hline
\end{tabular}

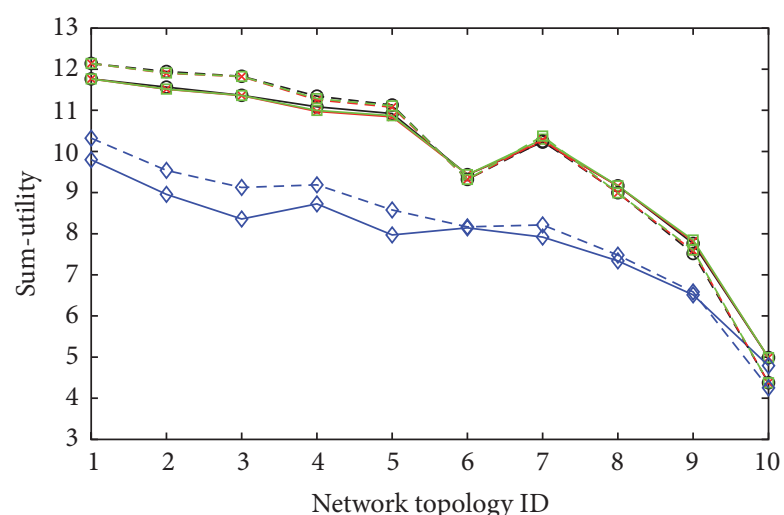

$\begin{array}{ll}\bullet \text { OPT, CONFIG1 } & -\ominus-\text { OPT, CONFIG2 } \\ \rightarrow \text { Multi, CONFIG1 } & -*-\text { Multi, CONFIG2 } \\ - \text { MultiR, CONFIG1 } & -\bullet-\text { MultiR, CONFIG2 } \\ \diamond \text { Greedy, CONFIG1 } & -\diamond-\text { Greedy, CONFIG2 }\end{array}$

FIgURE 3: Sum-utility for users-applications configurations CONFIG1 and CONFIG2, 18 users, scenario propitious, $w=0.5$, for the network topologies of Table 4 .
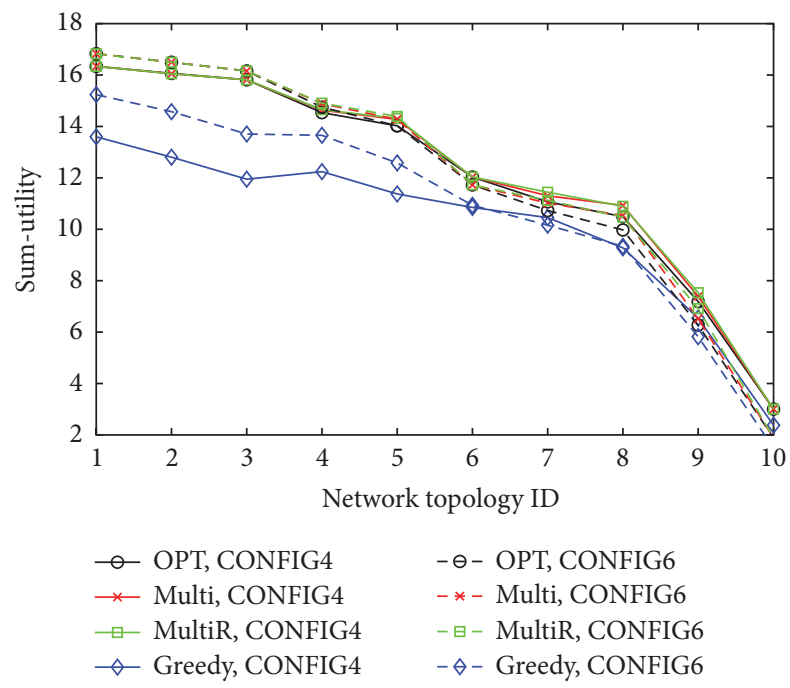

FIgURE 4: Sum-utility for users-applications configurations CONFIG4 and CONFIG6, 18 users, scenario propitious, $w=0.5$, for the network topologies of Table 4.

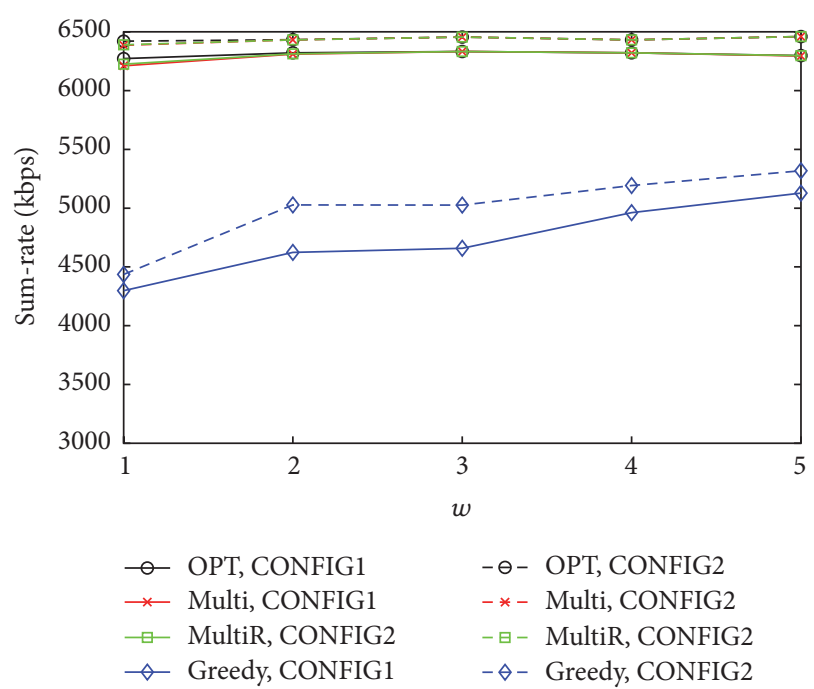

FIGURE 5: Sum-rate for users-applications configurations CONFIG1 and CONFIG2, 18 users, scenario propitious, network topology \#1.

negligible and even negative for network topologies 7-10. For other values of $w$, the curves exhibit a similar performance.

5.3. Bit Rate Performance. The performance in terms of sumrate is depicted in Figures 5-8, where we observe that an increase in the number of high-expectation users (CONFIG2 and CONFIG6) does not affect the total rate, except for the Greedy algorithm. Moreover, the sum-rate is almost constant irrespectively of the value of $w$, with the exception of the Greedy algorithm (see Figures 5 and 6). Figures 7 and 8 display the sum-rate as a function of the network topology (see Table 4), showing that the lower the capacity, the lower the sum-rate, as expected.

5.4. Impact of User's Profiles. We now evaluate the impact of the user's profiles. The figures plot data when the OPT solution is employed, as the other solutions exhibit similar performance. In Figure 9, we see how the network topology affects the performance in terms of mean utility per user: while for topologies with high capacity HEUs and MEUs clearly outperform LEUs, LEUs outperform MEUs for low 


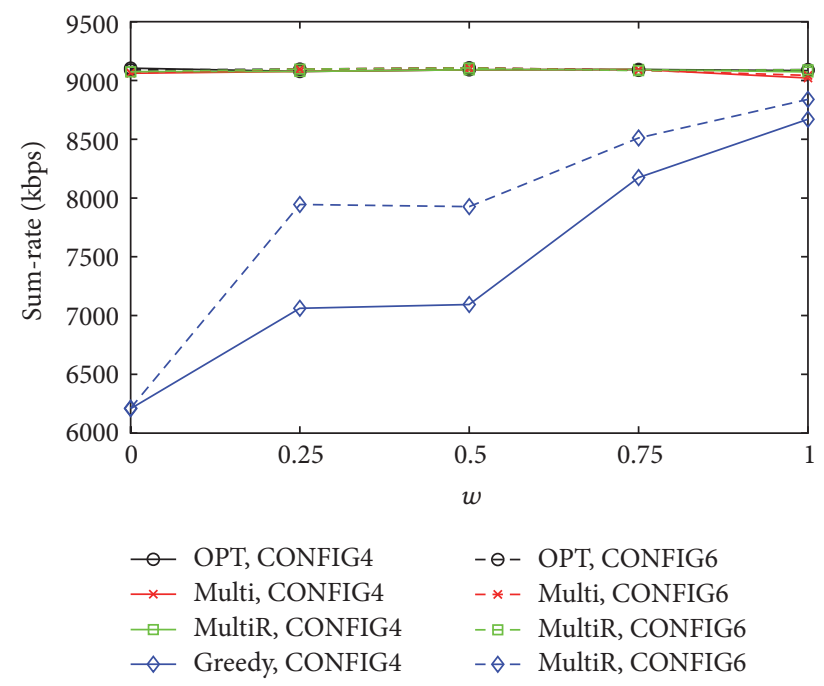

FIGURE 6: Sum-rate for users-applications configurations CONFIG4 and CONFIG6, 18 users, scenario propitious, network topology \#1.

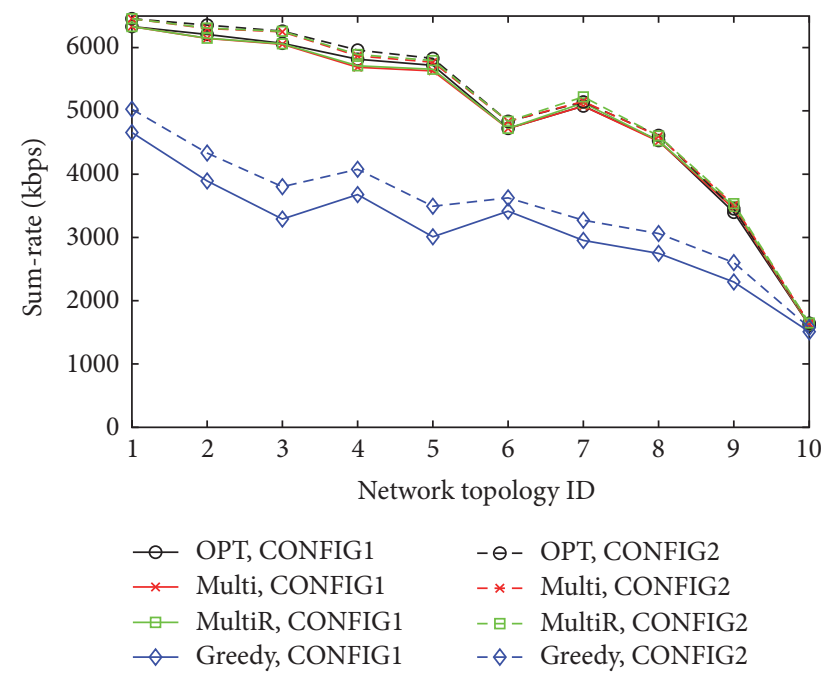

FIGURE 7: Sum-rate for users-applications configurations CONFIG1 and CONFIG2, 18 users, scenario propitious, $w=0.5$, for the network topologies of Table 4.

capacity and both MEUs and HEUs for network topology \#10. In Figure 10, we see that the value of $w$ impacts the mean utility of LEUs, while its effect is almost negligible for MEUs and HEUs. In Figures 11 and 12, we notice that there is no appreciable difference when the percentage of user types is significantly modified (CONFIG1 and CONFIG2, CONFIG4 and CONFIG6) for the different network topologies.

With respect to bit rate, as the optimization function does not directly focus on bit rate, variations in utility performance for different user's profiles may or may not translate into diversity in bit rate performance. As Figure 13 shows, there is no appreciable difference when the percentage of applications is similar (CONFIG1 and CONFIG2). However, if the percentage of application type is highly unbalanced

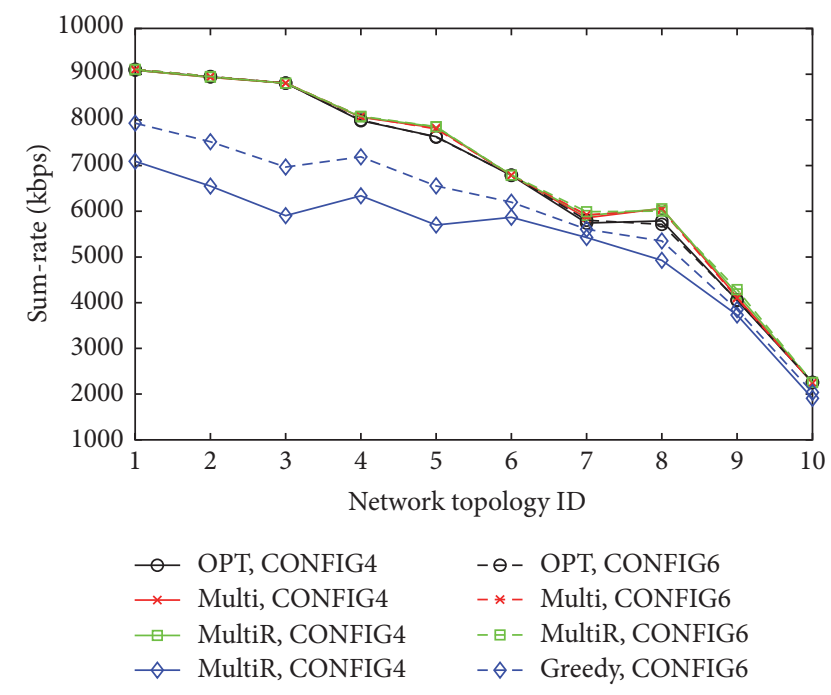

FIGURE 8: Sum-rate for users-applications configurations CONFIG4 and CONFIG6, 18 users, scenario propitious, $w=0.5$, for the network topologies of Table 4. Solution used: OPT.

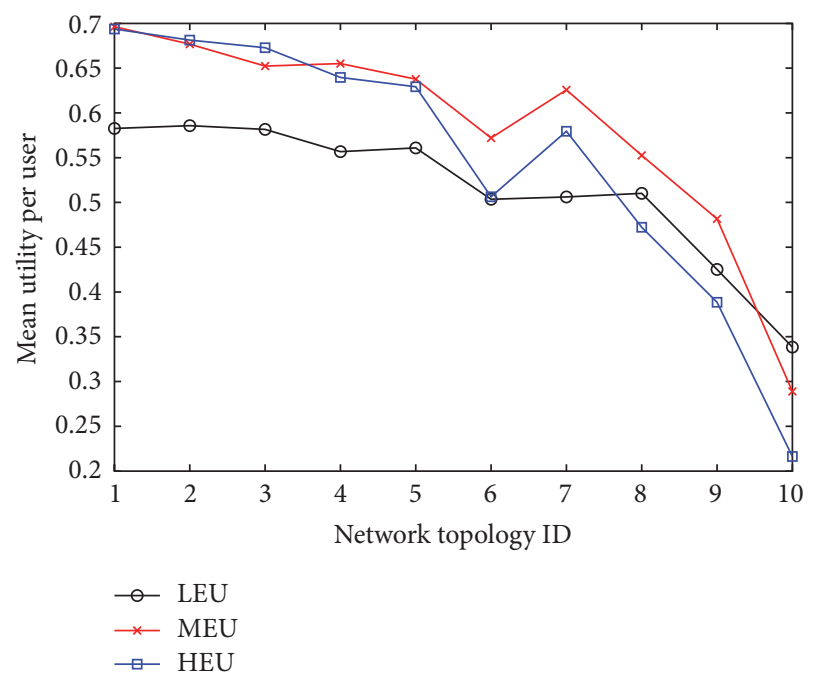

Figure 9: Mean utility per user with different user's profiles, 18 users, CONFIG1, scenario propitious, $w=0.5$, for the network topologies of Table 4. Solution used: OPT.

(CONFIG4 and CONFIG6), the percentage of user type influences the obtained bit rate. In this case (see Figure 14), the performances for LEUs, MEUs, and HEUs are dissimilar, especially for the low capacity network topologies.

5.5. BS Load. We now evaluate the BS load. We have observed that the variation of the user's profile has a negligible effect (for instance, in the case of CONFIG1 and CONFIG2 or CONFIG4 and CONFIG6), so CONFIG1 and CONFIG4 have been plotted as a representative case. We see that, depending on the network topology, the mean load varies (see Figure 15). The value of $w$ does not affect the mean BS load, irrespectively of the configuration or the network topology, as Figures 16 and 17 show. 


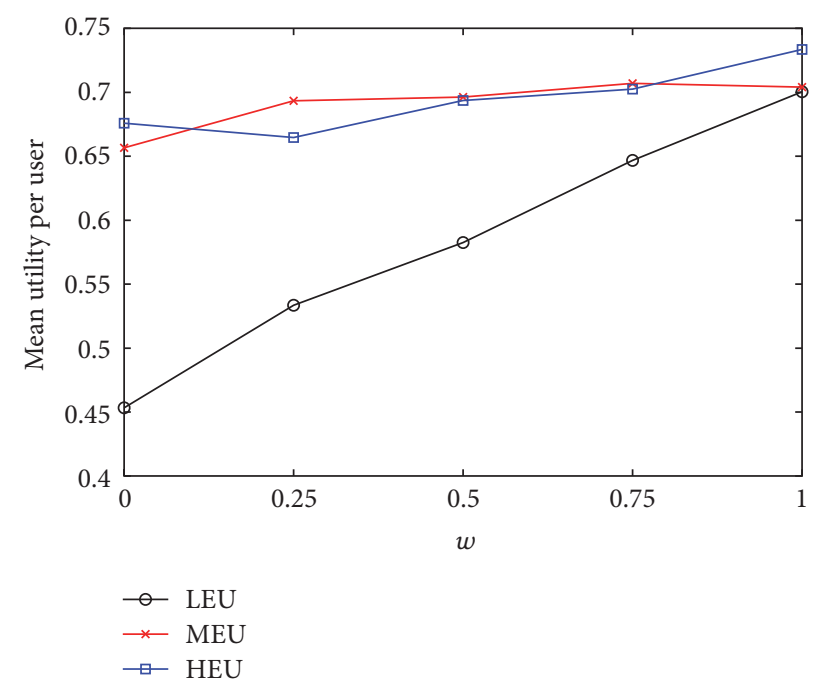

FIGURE 10: Mean utility per user with different user's profiles, 18 users, CONFIG1, scenario propitious, network topology \#1, as a function of $w$.

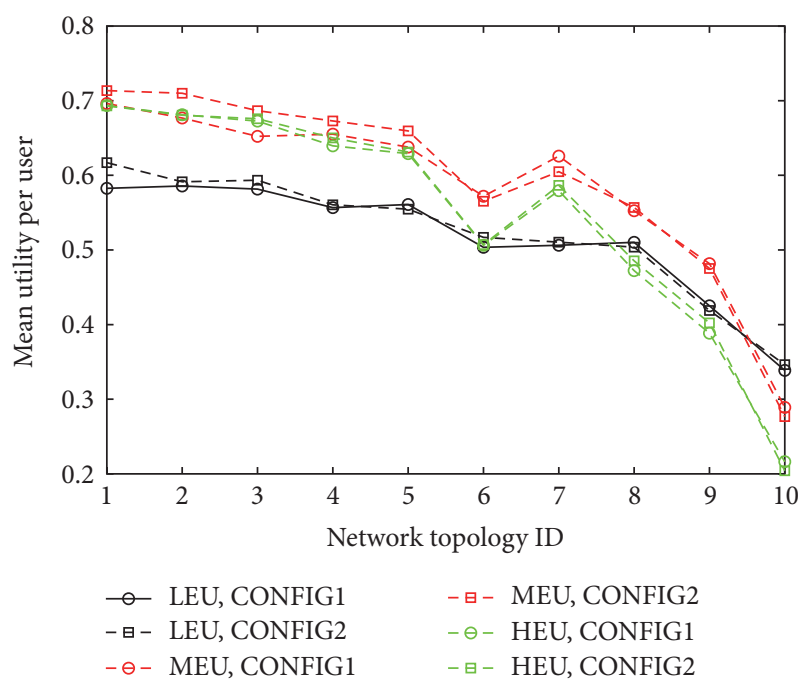

FIGURE 11: Mean utility per user with different user's profiles with CONFIG1 and CONFIG2, 18 users, scenario propitious, $w=0.5$, for the network topologies of Table 4.

5.6. Discussion. The results show that our proposal (OPT, Multi, and MultiR) outperforms the Greedy algorithm and that the OPT, Multi, and MultiR solutions achieve in practice the same performance, not only in terms of utility but also in terms of bit rate.

Regarding the number of users, we have run simulations for a number of users (applications) from 4 to 18 . We have observed that the number of users does not impact any of the metrics shown in the performance evaluation section, so we only present results for most unfavourable case (i.e., 18 applications).

We mention in the introduction of Section 5 that 3 scenarios have been considered for simulation (propitious,

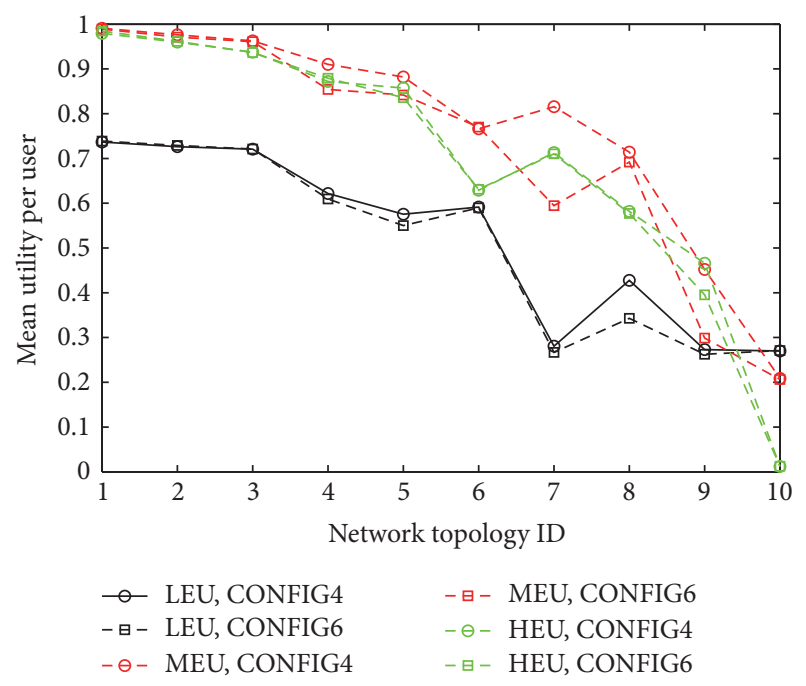

FIGURE 12: Mean utility per user with different user's profiles, 18 users with CONFIG4 and CONFIG6, scenario propitious, $w=0.5$, for the network topologies of Table 4.

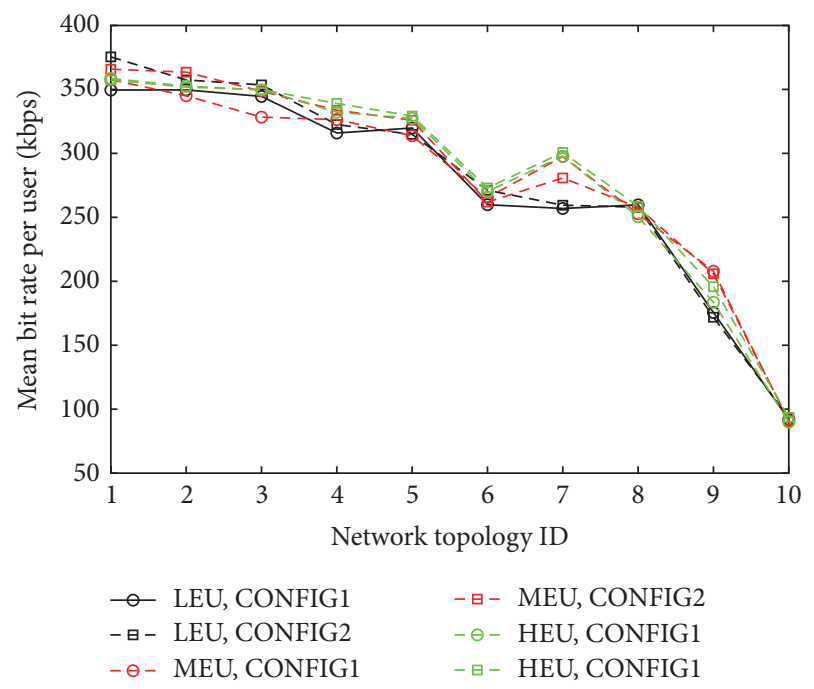

FIGURE 13: Mean bit rate per user with different user's profiles, 18 users with CONFIG1 and CONFIG1, scenario propitious, $w=0.5$, for the network topologies of Table 4 .

balanced, and ominous). However, the results for the balanced and ominous cases are akin to the propitious scenario except for the fact that the utility and bit rate values are lower. For this reason, we have not included results for the balanced and ominous scenarios.

\section{Conclusions}

In this work, we have formulated the NSP taking into account the fact that user terminals support multiple RATs and can be served simultaneously from diverse BSs. As not only user's satisfaction is important, provider's satisfaction has been included to build the objective utility function. We have seen 


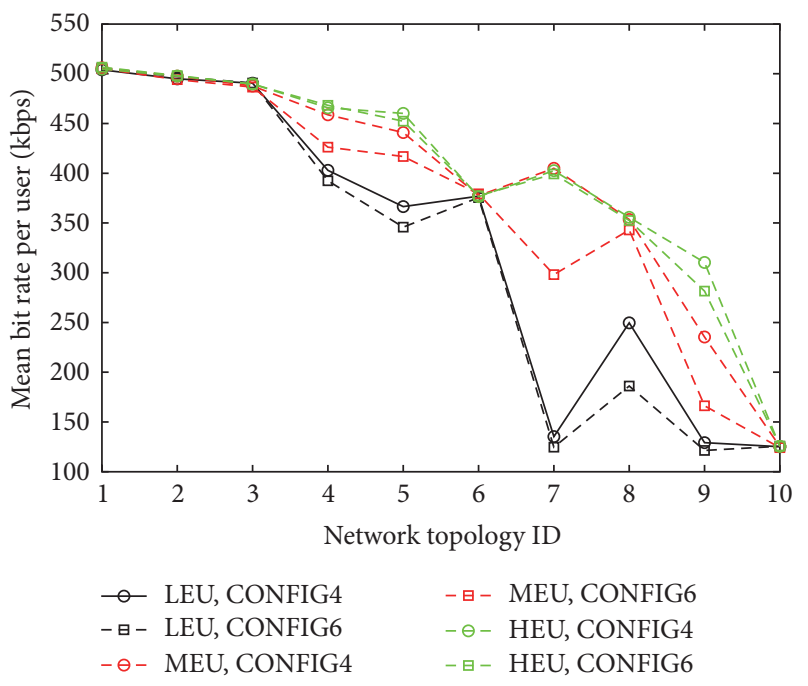

Figure 14: Mean bit rate per user with different user's profiles, 18 users with CONFIG4 and CONFIG6, scenario propitious, $w=0.5$, for the network topologies of Table 4 .

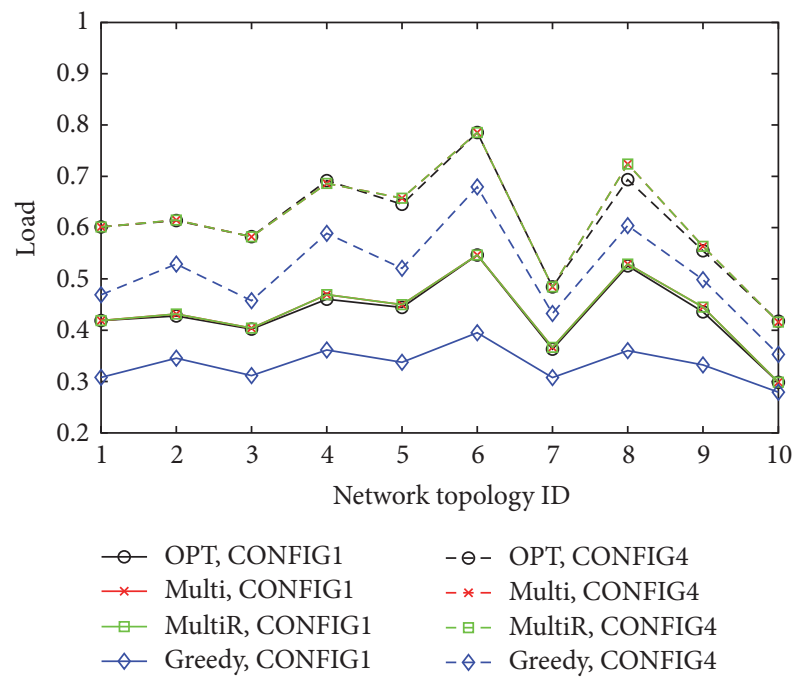

FIGURE 15: Mean load per BS (1 means 100\%), 18 users with CONFIG1 and CONFIG4, scenario propitious, $w=0.5$, for the network topologies of Table 4 .

that by means of parameter $w$ we can balance user's and provider's satisfaction.

As the NSP faces the optimization of the sum-utility, sum-rate performance is not meaningful from the user's profiles viewpoint. Nevertheless, the individual utility and rate are significantly influenced by the user's profiles and the percentage of user's types and application's types. Our simulations also show that the network topology impacts the system performance and that BS load can vary according to user's profiles.

In summary, we can conclude that, to design an effective association for the NSP, different factors must be taken into account, such as user's profiles, application's characteristics,

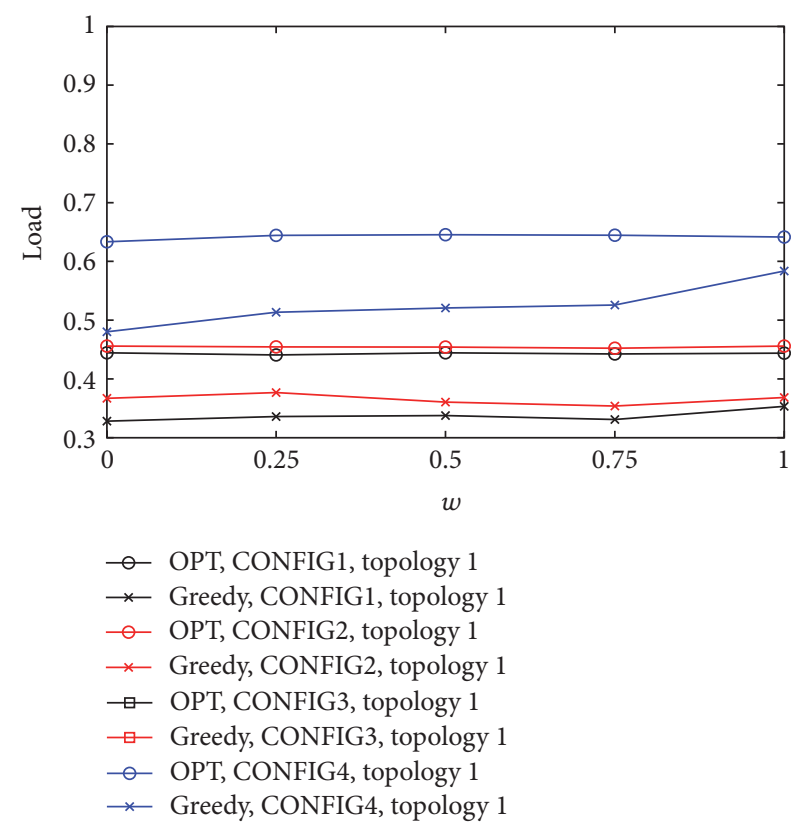

FIGURE 16: Mean load per BS (1 means 100\%), scenario propitious, for configurations of Table 3 , as a function of $w$, for network topology $\# 1$.

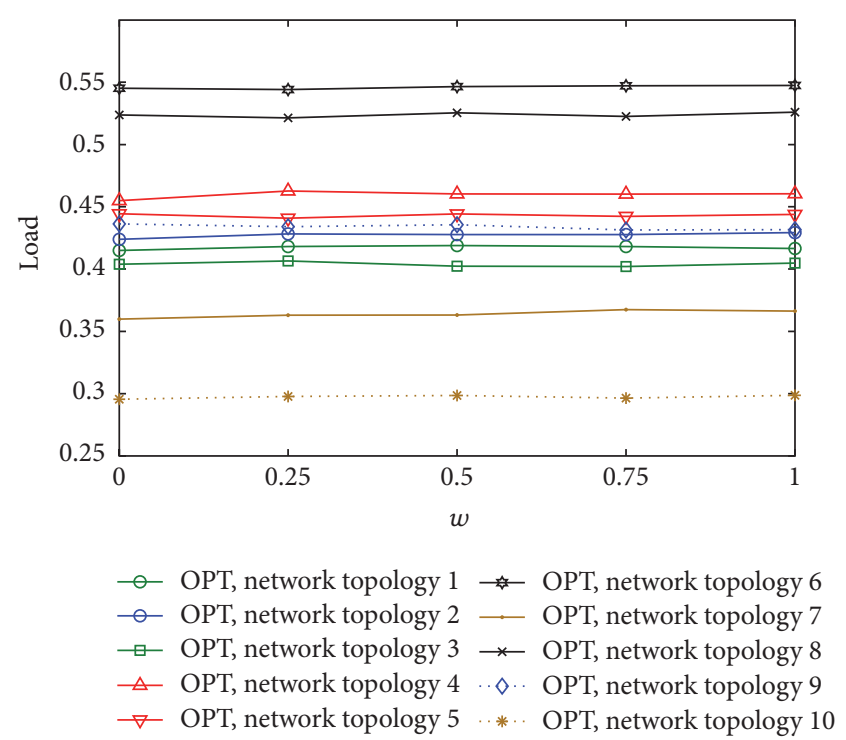

Figure 17: Mean load per BS (1 means 100\%), scenario propitious, for the network topologies of Table 4 , as a function of $w$, for CONFIG1.

the number and type of BSs that form the HetNet, and the operator's satisfaction.

\section{Competing Interests}

The authors declare that they have no competing interests. 


\section{Acknowledgments}

The work of J. J. Escudero-Garzás is funded in part by project ELISA (Enabling Technologies for Licensed and Unlicensed Shared Access Communications) TEC2014-59255-C3-3-R, Spanish Ministry of Economy and Competitiveness.

\section{References}

[1] J. G. Andrews, "Seven ways that hetnets are a cellular paradigm shift," IEEE Communications Magazine, vol. 51, no. 3, pp. 136144, 2013.

[2] S. Glisic, "Heterogeneous networks," in Advanced Wireless Networks: Technology and Business Models, chapter 11, pp. 402445, John Wiley \& Sons, Chichester, UK, 3rd edition, 2016.

[3] Q. Song and A. Jamalipour, "An adaptive quality-of-service network selection mechanism for heterogeneous mobile networks," Wireless Communications and Mobile Computing, vol. 5, no. 6, pp. 697-708, 2005.

[4] S.-Y. Lien, S.-M. Cheng, S.-Y. Shih, and K.-C. Chen, "Radio resource management for QoS guarantees in cyber-physical systems," IEEE Transactions on Parallel and Distributed Systems, vol. 23, no. 9, pp. 1752-1761, 2012.

[5] J. Mitola, J. Guerci, J. Reed et al., "Accelerating 5G QoE via public-private spectrum sharing," IEEE Communications Magazine, vol. 52, no. 5, pp. 77-85, 2014.

[6] O. E. Falowo and H. A. Chan, "Heuristic RAT selection policy to minimize call blocking probability in next generation wireless networks," Wireless Communications and Mobile Computing, vol. 10, no. 2, pp. 214-229, 2010.

[7] M. Lopez-Benitez and J. Gozalvez, "Common radio resource management algorithms for multimedia heterogeneous wireless networks," IEEE Transactions on Mobile Computing, vol. 10, no. 9, pp. 1201-1213, 2011.

[8] O. E. Falowo and H. A. Chan, "Dynamic RAT selection for multiple calls in heterogeneous wireless networks using group decision-making technique," Computer Networks, vol. 56, no. 4, pp. 1390-1401, 2012.

[9] Q.-T. Nguyen-Vuong, N. Agoulmine, E. H. Cherkaoui, and L. Toni, "Multicriteria optimization of access selection to improve the quality of experience in heterogeneous wireless access networks," IEEE Transactions on Vehicular Technology, vol. 62, no. 4, pp. 1785-1800, 2013.

[10] Y.-S. Liou, R.-H. Gau, and C.-J. Chang, "A bargaining game based access network selection scheme for HetNet," in Proceedings of the 1st IEEE International Conference on Communications (ICC '14), pp. 4888-4893, IEEE, Sydney, Australia, June 2014.

[11] P. Kosmides, A. Rouskas, and M. Anagnostou, "Utility-based RAT selection optimization in heterogeneous wireless networks," Pervasive and Mobile Computing, vol. 12, pp. 92-111, 2014.

[12] A. Kumar, R. K. Mallik, and R. Schober, "A probabilistic approach to modeling users' network selection in the presence of heterogeneous wireless networks," IEEE Transactions on Vehicular Technology, vol. 63, no. 7, pp. 3331-3341, 2014.

[13] J. Konka, I. Andonovic, C. Michie, and R. Atkinson, "Auctionbased network selection in a market-based framework for trading wireless communication services," IEEE Transactions on Vehicular Technology, vol. 63, no. 3, pp. 1365-1377, 2014.

[14] N. Sui, D. Zhang, W. Zhong, and C. Wang, "Network selection for heterogeneous wireless networks based on multiple attribute decision making and Evolutionary Game Theory," in Proceedings of the 25th Wireless and Optical Communication Conference (WOCC '16), pp. 1-5, May 2016.

[15] L. Wang and G.-S. G. S. Kuo, "Mathematical modeling for network selection in heterogeneous wireless networks-a tutorial," IEEE Communications Surveys and Tutorials, vol. 15, no. 1, pp. 271-292, 2013.

[16] A. Sgora, C. A. Gizelis, and D. D. Vergados, "Network selection in a WiMAXWiFi environment," Pervasive and Mobile Computing, vol. 7, no. 5, pp. 584-594, 2011.

[17] M. Ismail and W. Zhuang, "A distributed multi-service resource allocation algorithm in heterogeneous wireless access medium," IEEE Journal on Selected Areas in Communications, vol. 30, no. 2, pp. 425-432, 2012.

[18] O. Galinina, A. Pyattaev, S. Andreev, M. Dohler, and Y. Koucheryavy, "5G multi-RAT LTE-WiFi ultra-dense small cells: performance dynamics, architecture, and trends," IEEE Journal on Selected Areas in Communications, vol. 33, no. 6, pp. 12241240, 2015.

[19] M. El Helou, M. Ibrahim, S. Lahoud, K. Khawam, D. Mezher, and B. Cousin, "A network-assisted approach for RAT selection in heterogeneous cellular networks," IEEE Journal on Selected Areas in Communications, vol. 33, no. 6, pp. 1055-1067, 2015.

[20] J. Kim, J. H. Lee, H. Lee, T. Kwon, and Y. Choi, "A network selection scheme for multicast applications in wireless network convergence," Wireless Communications and Mobile Computing, vol. 12, no. 11, pp. 999-1012, 2012.

[21] G. Lim, C. Xiong, L. J. Cimini, and G. Y. Li, "Energy-efficient resource allocation for OFDMA-based multi-RAT networks," IEEE Transactions on Wireless Communications, vol. 13, no. 5, pp. 2696-2705, 2014.

[22] G. Yu, Y. Jiang, L. Xu, and G. Y. Li, "Multi-objective energyefficient resource allocation for multi-RAT heterogeneous networks," IEEE Journal on Selected Areas in Communications, vol. 33, no. 10, pp. 2118-2127, 2015.

[23] J. Miao, Z. Hu, K. Yang, C. Wang, and H. Tian, "Joint power and bandwidth allocation algorithm with QoS support in heterogeneous wireless networks," IEEE Communications Letters, vol. 16, no. 4, pp. 479-481, 2012.

[24] B. Fu, Z. Wei, X. Yan, K. Zhang, Z. Feng, and Q. Zhang, "A game-theoretic approach for bandwidth allocation and pricing in heterogeneous wireless networks," in Proceedings of the IEEE Wireless Communications and Networking Conference (WCNC '15), pp. 1684-1689, IEEE, New Orleans, La, USA, March 2015.

[25] Q. Ye, B. Rong, Y. Chen, M. Al-Shalash, C. Caramanis, and J. G. Andrews, "User association for load balancing in heterogeneous cellular networks," IEEE Transactions on Wireless Communications, vol. 12, no. 6, pp. 2706-2716, 2013.

[26] E. Björnson, E. A. Jorswieck, M. Debbah, and B. Ottersten, "Multiobjective signal processing optimization: the way to balance conflicting metrics in 5G systems," IEEE Signal Processing Magazine, vol. 31, no. 6, pp. 14-23, 2014.

[27] Y. Liu, Z. Chen, L. T. Yang, T. Li, and X. Lv, "User preference heterogeneous network selection in less subjective ways," Wireless Personal Communications, vol. 76, no. 4, pp. 813-828, 2014.

[28] R. Wang, X. Wang, F. Li, and M. Huang, "An IEEE 802.21 based heterogeneous access network selection mechanism," in Advanced Intelligent Computing Theories and Applications, D.-S. Huang and K. Han, Eds., vol. 9227 of Lecture Notes in Computer Science, pp. 574-585, Springer International, Berlin, Germany, 2015. 
[29] J. J. Escudero-Garzás and A. García-Armada, "Game theory for PHY layer and MAC sublayer in wireless telecommunications," in Networking and Telecommunications: Concepts, Methodologies, Tools, and Applications, Information Resources Management Association (IRMA), Ed., chapter 109, pp. 16981713, IGI Global, Hershey, NY, USA, 2010.

[30] R. Trestian, O. Ormond, and G.-M. Muntean, "Game theorybased network selection: solutions and challenges," IEEE Communications Surveys and Tutorials, vol. 14, no. 4, pp. 1212-1231, 2012.

[31] Z. Du, Q. Wu, P. Yang, Y. Xu, and Y.-D. Yao, "User-demandaware wireless network selection: a localized cooperation approach," IEEE Transactions on Vehicular Technology, vol. 63, no. 9, pp. 4492-4507, 2014.

[32] Y. Cao, D. Duan, X. Cheng, L. Yang, and J. Wei, "Dynamic network selection in HetNets: a social-behavioral (SoBe) approach," in Proceedings of the IEEE Global Communications Conference (GLOBECOM '14), pp. 4653-4658, Austin, Tex, USA, December 2014.

[33] M. Peng, X. Xie, Q. Hu, J. Zhang, and H. V. Poor, "Contractbased interference coordination in heterogeneous cloud radio access networks," IEEE Journal on Selected Areas in Communications, vol. 33, no. 6, pp. 1140-1153, 2015.

[34] F. Agnelli, G. Albasini, I. Bietti et al., "Wireless multi-standard terminals: system analysis and design of a reconfigurable RF front-end," IEEE Circuits and Systems Magazine, vol. 6, no. 1, pp. 38-57, 2006.

[35] Q.-T. Nguyen-Vuong, Y. Ghamri-Doudane, and N. Agoulmine, "On utility models for access network selection in wireless heterogeneous networks," in Proceedings of the IEEE Network Operations and Management Symposium (NOMS '08), pp. 144151, April 2008.

[36] C. Y. Wong, R. S. Cheng, K. B. Letaief, and R. D. Murch, "Multiuser OFDM with adaptive subcarrier, bit, and power allocation," IEEE Journal on Selected Areas in Communications, vol. 17, no. 10, pp. 1747-1758, 1999.

[37] L. A. Wolsey, Integer Programming, Wiley-Interscience Series in Discrete Mathematics and Optimization, John Wiley \& Sons, New York, NY, USA, 1998.

[38] R. H. Byrd, M. E. Hribar, and J. Nocedal, "An interior point algorithm for large-scale nonlinear programming," SIAM Journal on Optimization, vol. 9, no. 4, pp. 877-900, 1999.

[39] N. A. Prabha, G. V. Sagar, and V. Manikandan, "Performance analysis of a hybrid multiple access technique in LTE system," in Proceedings of the 2nd International Conference on Communication and Signal Processing (ICCSP '13), pp. 648-652, Andhra Pradesh, India, April 2013. 


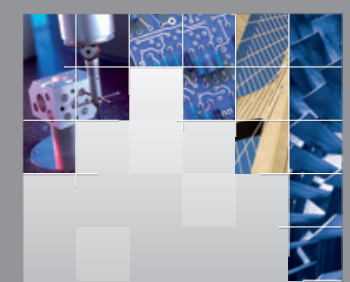

\section{Enfincering}
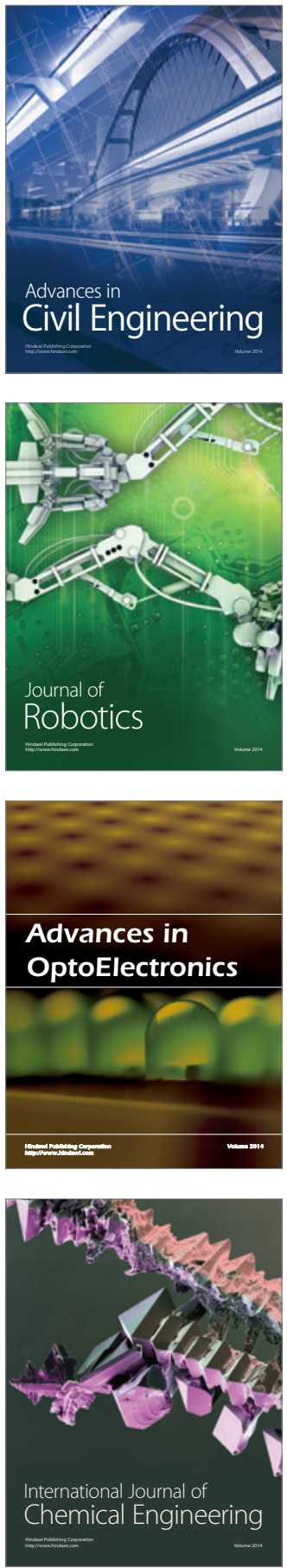

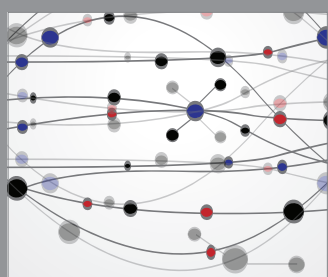

The Scientific World Journal

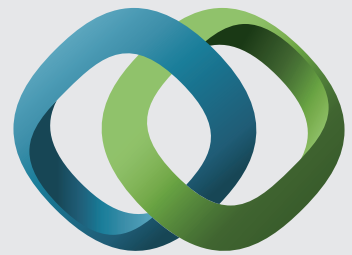

\section{Hindawi}

Submit your manuscripts at

https://www.hindawi.com
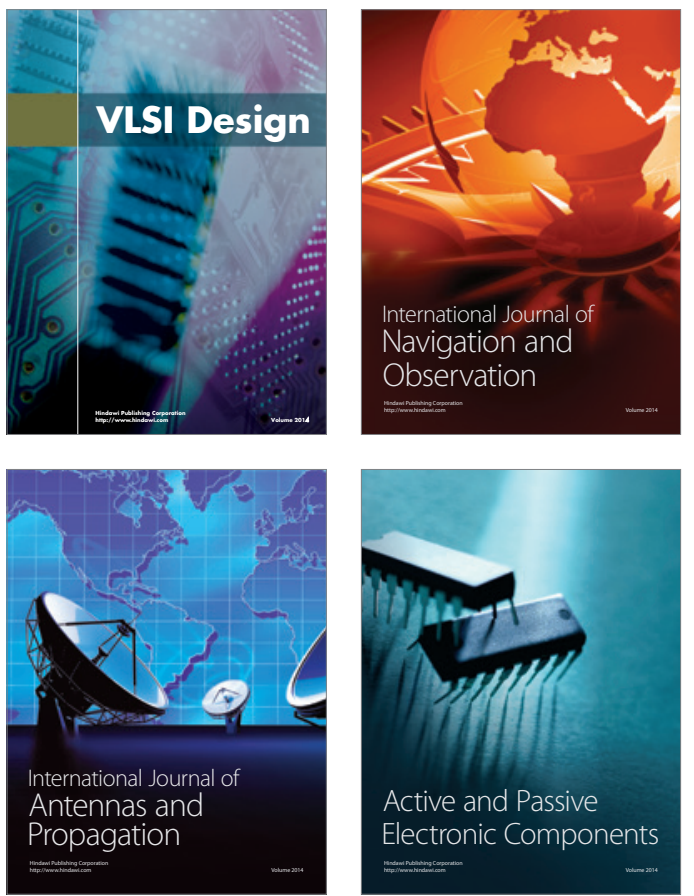
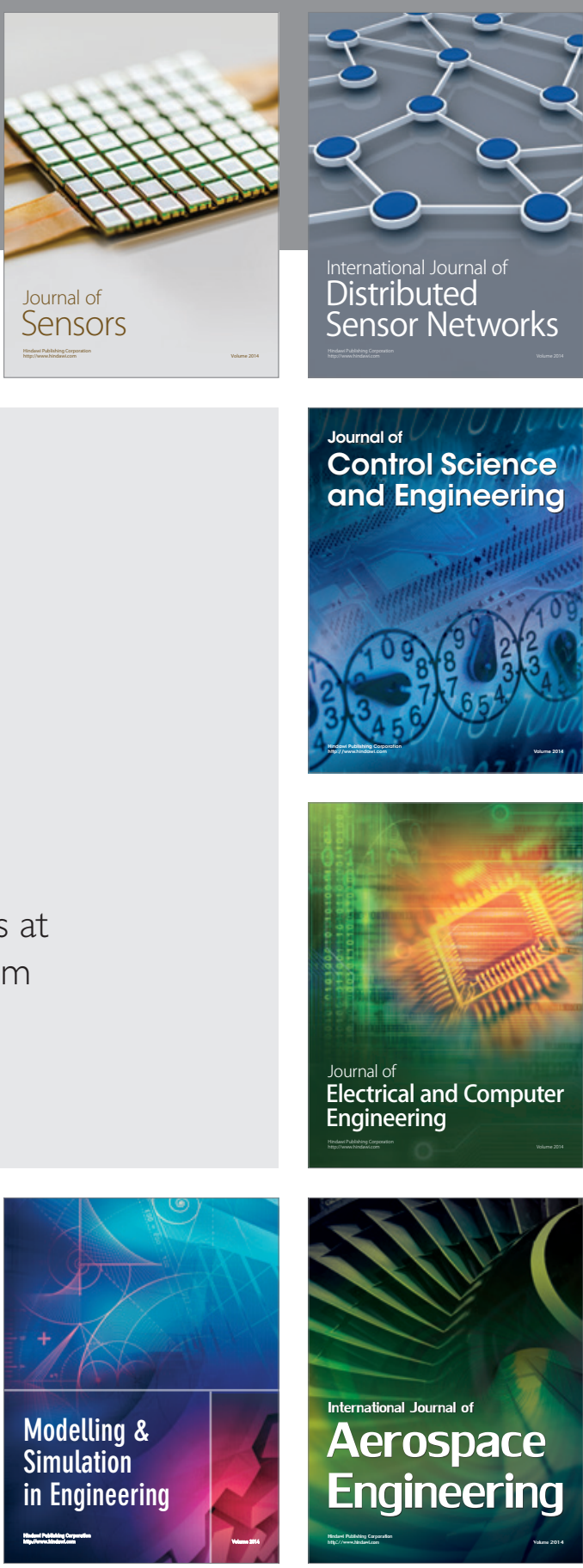

International Journal of

Distributed

Sensor Networks

$-$

Joumal of

Control Science

and Engineering
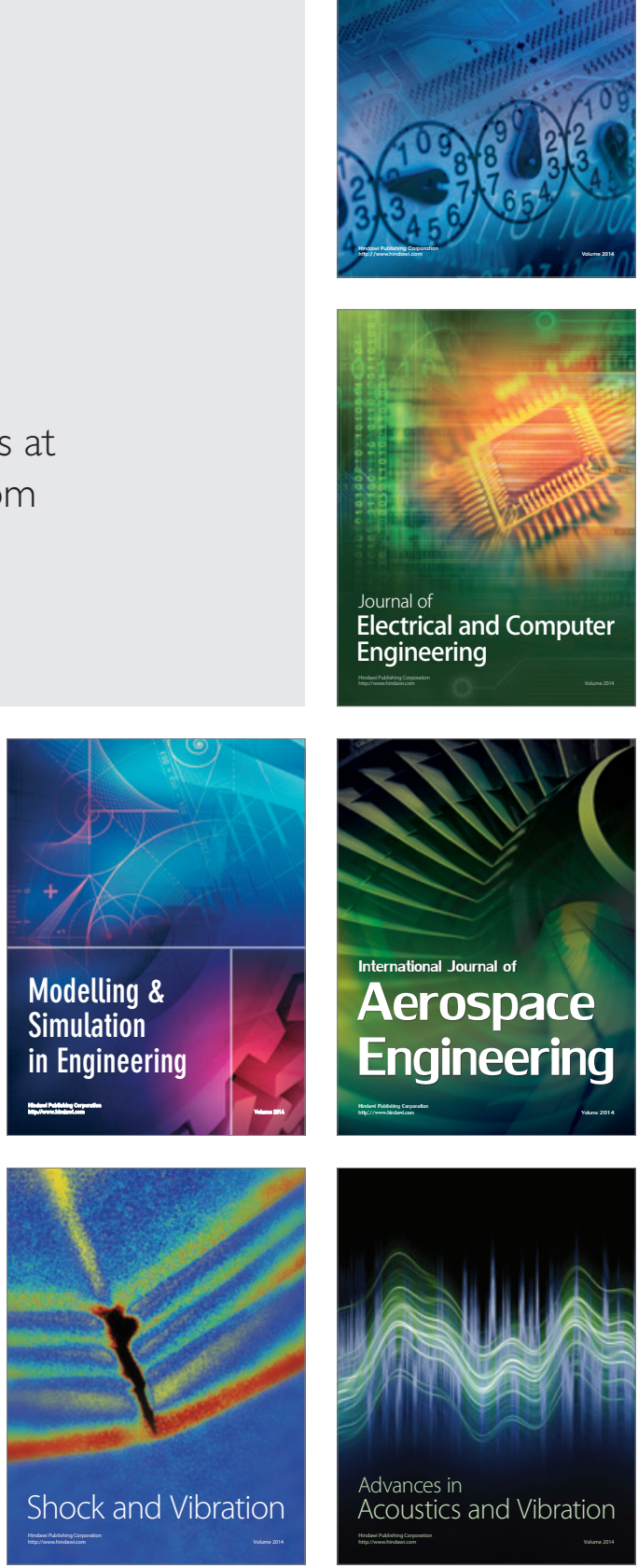